\title{
Wie aus Familiengeschichten sorgsame Landwirtschaft entsteht
}

Andrea Heistinger

Wie in der Einleitung ausführlich beschrieben, ist der Handlungsdruck innerhalb der Agrar- und Ernährungswirtschaft geradezu unvorstellbar groß. Die vorindustrielle bäuerliche Landwirtschaft gibt es nicht mehr, ${ }^{1}$ die industrielle konventionelle Landwirtschaft hat sich als nicht zukunftsfähig erwiesen und selbst der biologische Landbau hat in den letzten 20 Jahren unter einem enormen sozialen und ökonomischen Konventionalisierungsdruck viel seiner Innovationskraft und Bedeutung als existenzsichernde Alternative für kleinere und mittlere landwirtschaftliche Betriebe verloren. Dazu auch eine aktuelle Zahl: Die ökologisch wirtschaftenden Testbetriebe Deutschlands haben im Wirtschaftsjahr 2018/2019 im Durchschnitt ein Einkommen von ca. $€ 38.000$ pro Arbeitskraft erzielt. Das sind zwar um $€ 10.000$ mehr als der Durchschnitt der konventionellen Betriebe, aber um $6 \%$ weniger als im Vergleich zum Vorjahr. ${ }^{2}$

Die globale Land- und Ernährungswirtschaft bedarf einer grundlegenden Transformation. Dabei geht es nicht um eine große Transformation, sondern um viele lokale und überregionale Transformationsprozesse, die ineinander greifen und sich gegenseitig verstärken können: Transformationsprozesse sowohl von politischer Seite, ${ }^{3}$ wie

1 Vgl. Hiß u.a. 2018.

2 Thünen Institut 2020.

3 Siehe dazu das im Mai 2020 veröffentlichte Strategiepapier der EU-Kommission »From Farm to Fork«. Es skizziert den notwendigen Wandel zu einem 
auch der Ernährungsgewohnheiten, der agrarischen Ausbildungen, direkt auf den Betrieben sowie neue Formen von Beziehungen, die Menschen zueinander und $\mathrm{zu}$ anderen Lebewesen unseres Planeten haben.

Die sozialwissenschaftliche Transformationsforschung definiert Transformation als eine besondere Form von Wandel. Denn »Neues und mithin Unvertrautes wird in Gesellschaften ständig verhandelt, ausgehandelt und schließlich in Vertrautes übergeführt. ${ }^{4}$ Der Unterschied zwischen Wandel und Transformation liege darin, dass »Umwälzungsprozesse am Wirken sind. (...) Wo Umwälzungen im Gange sind, wird Vertrautes brüchig, werden Routinen des Alltags in Frage gestellt und erhebliche Bestände des alltäglichen Orientierungswissens fragwürdig. ${ }^{5}$ Dies betrifft sowohl akademische und in Schulsystemen vermittelte Wissensbestände - zum Beispiel, wie man einen Betrieb ökonomisch erfolgreich führt - wie auch bislang bewährte Praxis-Erfahrungen, wie Kulturpflanzen angebaut oder wie bestimmte Betriebszweige gehandhabt werden. Und ebenso Fragen des sozialen Zusammenlebens. Letzteres wird am deutlichsten in der Öffentlichkeit wahrgenommen durch den Umstand, dass es für

»nachhaltigen Lebensmittelsystem «und benennt konkrete Ziele, um dies zu erreichen. So soll die Abhängigkeit von Pestiziden und antimikrobiellen Mitteln verringert, der übermäßige Einsatz von Düngemitteln reduziert, der Ökologische Landbau ausgedehnt, der Tierschutz verbessert und der Verlust an biologischer Vielfalt rückgängig gemacht werden. Auslöser für die klaren Worte und Ziele des Papers war vermutlich auch die COVID-19 Pandemie: „Die COVID_19Pandemie hat ein Schlaglicht auf die Bedeutung eines belastbaren und resilienten Lebensmittelsystems geworfen, das unter jeglichen Umständen weiter funktioniert und in der Lage ist, die Bürgerinnen und Bürger in ausreichendem Maße mit erschwinglichen Lebensmitteln zu versorgen. Sie hat uns auch die Wechselwirkungen zwischen unserer Cesundheit, unseren Ökosystemen, Versorgungsketten, Verbrauchsmustern und den Belastungsgrenzen unseres Planeten sehr bewusst gemacht. Es liegt auf der Hand, dass wir viel mehr tun müssen, um uns und den Planeten gesund zu hallten. «European Commission 2020, S. 2.

$4 \quad$ Hildenbrand 2015, S. 232.

$5 \quad$ Hildenbrand 2015, S. 231. 
junge männliche Hoferben zunehmend schwieriger geworden ist, eine Partner*in $\mathrm{zu}$ finden. Die geschlossenen Heiratskreise, die bis vor 20 oder 30 Jahren hier Orientierung und Sozialisation boten, haben sich vielerorts fast vollständig aufgelöst.

Die sinnverstehende Sozialforschung geht davon aus, dass jeder Wandel durch soziale Interaktionen hervorgebracht und es die - handelnden - Menschen sind, die diesen Wandel mit Bedeutung versehen. Gegenstand der sinnverstehenden Sozialforschung ist daher jener

»Prozess, innerhalb dessen das Verhältnis von Vertrautem und Fremdem in der sozialen Wirklichkeit für die Akteure in weiten Bereichen fragwürdig geworden ist, gleichzeitig aber ein Handlungsdruck besteht, Vertrautheit und Routine wiederherzustellen und ihr einen Sinn zu verleihen. ${ }^{6}$

Viele Menschen, die in der Landwirtschaft tätig waren, haben in den letzten Jahren und Jahrzehnten die Entscheidung getroffen, ihre Betriebe zu schließen und einer Erwerbsarbeit außerhalb der Landwirtschaft nachzugehen. Vor allem für junge Menschen war die soziale Wirklichkeit des Berufs Bauer/Bäuerin ${ }^{7}$ fragwürdig geworden und der ökonomische Druck zu groß, um den Beruf und das Familienunternehmen ihrer Eltern zu übernehmen. Sie schlossen die Höfe, die oft seit vielen Generationen als landwirtschaftliche Betriebe bewirtschaftet worden waren. Meist wird dies als "Betriebsaufgabe« bezeichnet. Dieser Begriff ist negativ konnotiert, im "Aufgeben « schwingt auch ein individuelles Versagen oder ein persönliches »Es ist mir nicht gelungen« mit. Mit der Schließung der Betriebe geht auch ein Wandel des beruflichen Habitus, der sozialen Praktiken innerhalb der Familie, die häufig dann keine Unternehmerfamilie/kein Familienunternehmen mehr ist,

6 Hildenbrand 2015, S. 232.

7 Dasselbe trifft für viele andere Berufe zu: Auch viele Wirte und Wirtinnen und Unternehmen in der Lebensmittelverarbeitung - Bäckereien, Fleischereien sowie Händler*innen im Lebensmittelbereich haben in den letzten Jahrzehnten ihre Betriebe geschlossen. Die Anzahl der Familienunternehmen ist auch in diesen Branchen gesunken. 
einher. Die Handlungsmuster, die die Familie prägen, ändern sich. ${ }^{8}$ Im Einzelnen mag dies ein Prozess des Wandels (im oben beschriebenen Sinn) sein. Gesamtgesellschaftlich ist dies ein großer Transformationsprozess: Jene regionalen Wirtschaftssysteme, die notwendig sind, um die lokalen Ressourcen (Boden, Wasser, Sonne, Luft) in Lebensmittel für jene Menschen zu transformieren, die in der Region leben, funktionierten nur mehr zu einem kleinen Anteil.

\section{Ausstieg aus der Landwirtschaft}

Die große gesellschaftliche Entwicklung ist die des Ausstiegs aus der Landwirtschaft. Zu diesem Ergebnis kommt man recht nüchtern, wenn man sich die Zahlen der landwirtschaftlichen Betriebe und ihre Abnahme über die letzten Jahrzehnte vor Augen führt. ${ }^{9}$ Das Phänomen wird als Strukturwandel in der Landwirtschaft bezeichnet oder als Prozess beschrieben: Wachsen oder Weichen. Dieses Wortbild bezieht sich auf die Zunahme der Hektar-Anzahl, die ein Betrieb bewirtschaftet (oder die Zunahme der Anzahl der auf dem Betrieb gehaltenen Tiere), bei einer gleichzeitigen Aufgabe anderer Betriebe. Doch trotz des Strukturwandels und trotz massiver Förderungen von Seiten der EU im Rahmen der Gemeinsamen Agrarpolitik (GAP) hat sich die Einkommenssituation in der Landwirtschaft in den letzten Jahren verschlechtert: Die Nettowertschöpfung in der österreichischen Landwirtschaft hat zwischen den Jahren 2000 bis 2015 um mehr als ein Viertel abgenommen. ${ }^{10} \mathrm{EU}$ -

Siehe dazu das Thema Handeln und Familienmuster im Kapitel »Sinn verstehen durch rekonstruktive Genogrammarbeit«.

9 In der EU schlossen zwischen den Jahren 2000 bis 2013 ein Viertel aller Landwirtschaftlichen Betriebe. Vgl. Agrar-Atlas, siehe www.boell.de (Zugriff am 22. Juni 2020). Im Jahr 1999 gab es in Österreich 217.508 Land- und Forstwirtschaftliche Betriebe im Jahr 2010 173.317, im Jahr 2013 166.317. Vgl. Landwirtschaftskammer Österreich 2016, S. 11.

10 Dies hat in erster Linie damit zu tun, dass die Ausgaben für Betriebsmittel und Dienstleistungen deutlich zugenommen haben, sowie die Ausgaben für Pachtzahlungen und Löhne, siehe Landwirtschaftskammer Österreich 2016, S. 8. 
weit betrachtet verdient ein Landwirt/eine Landwirtin durchschnittlich derzeit nur etwa halb so viel wie ein Arbeitnehmer/eine Arbeitnehmerin. ${ }^{11}$ Auch diese Einkommenssituation hat dazu geführt, dass viele Söhne und Töchter landwirtschaftlicher Familien es bevorzugen, Betriebe nicht zu übernehmen und einen Beruf außerhalb der Landwirtschaft zu erlernen und diesen als Vollzeitberuf auszuüben. Mit dieser Entwicklung einher geht in vielen Regionen ein Rückgang der Bevölkerung in ländlichen Regionen. Die regionale Bevölkerungsprognose 2030/2050 der Österreichischen Raumordnungskonferenz (ÖROK) zeigt, dass der demografische Wandel und der Bevölkerungsrückgang in vielen österreichischen Regionen ein zentrales Thema sein wird. War bereits in den letzten 50 Jahren die Geburtenbilanz in vielen ländlichen Regionen negativ, hat sich die Landflucht seit den 2000 er-Jahren nochmals verstärkt: $30 \%$ aller politischen Bezirke in Österreich und mehr als $40 \%$ aller Gemeinden sind mit einem Bevölkerungsrückgang konfrontiert. ${ }^{12}$ Für unsere Forschungsregionen - die Oststeiermark und das Waldviertel - lässt sich sagen, dass in der Oststeiermark seit den 1990er Jahren ein Bevölkerungsrückgang zu verzeichnen ist, während im Waldviertel dieser Prozess bereits in den 1960er Jahren begann. ${ }^{13}$ In erster Linie sind es jungen Frauen, die vom Land in die Stadt gehen und nicht mehr - zum Beispiel in der Lebensphase der Familiengründung zurückkehren. ${ }^{14}$ Dabei hat der »Exodus« der jungen Frauen vom Land nicht nur wirtschaftliche, sondern auch »lebensweltliche Gründe«, wie die Agrarsoziologin Theresia Oedl-Wieser beschreibt. ${ }^{15}$ Insbesondere weist sie auf ein Festhalten an starren traditionellen Geschlechterrollen hin, die die Entwicklungsperspektiven für Frauen am Land stark einschränken. Die Raumplanerinnen Gerhild Weber und Tatjana Fischer weisen darauf hin, dass mit der selektiven Abwanderung von Frauen eine negative Entwicklungsspirale für ländliche Regionen in Gang gesetzt

\footnotetext{
11 European Commission 2020, S. 2.

12 Dax u.a. 2016, S. 5.

13 Dax u.a. 2016, S. 22.

14 Oedl-Wieser 2017.

15 Oedl-Wieser 2017, S. 5.
} 
wird, die nicht nur einen Verlust an Know-How, an Tatkraft und Reproduktionsfähigkeit mit sich bringt, sondern letztlich auch dazu führt, dass die Bevölkerung überaltert, die Finanzkraft der öffentlichen Hand sinkt, lokale Infrastruktur abgebaut wird, Dörfer und ländliche Regionen ihr Attraktivität verlieren, das Gemeinschaftsleben stirbt, sich in diesen Regionen eine »No Future-Stimmung « breit macht und all dies die Dynamik einer weiteren Abwanderung stärkt. ${ }^{16}$

\section{Einstieg in die Landwirtschaft}

All diesen Faktoren zum Trotz wollen immer mehr junge Menschen sowie Menschen, die bereits einen beruflichen Werdegang in einer anderen Branche eingeschlagen hatten, in die Landwirtschaft einsteigen. ${ }^{17}$ Vor dem Hintergrund all dieser Entwicklungen ist insbesondere die Frage interessant, wer die Menschen sind, die landwirtschaftliche Betriebe neu gründen. Ausgehend von der These, dass es auch in den untersuchten Regionen Menschen gibt, die neu in die Landwirtschaft einsteigen und dass dieser Einstieg Merkmal eines gesellschaftlichen Transformationsprozesses ist, stellt unsere Forschung die Frage: Wer sind die Menschen, die diese Transformationsprozesse vorantreiben? Wie gelingt es ihnen Wirtschaftsformen zu finden, die für sie die biologische Landwirtschaft unter dem Druck des agrarindustriell organisierten Marktes wirtschaftlich erfolgreich macht? Welche Ressourcen bringen sie dafür aus ihren Familien-Systemen mit? Welche Familienmuster sind dafür hilfreich?

Insbesondere fragt die vorliegende Studie nach resilienten Formen und Handlungsmustern einer ökologischen Landwirtschaft, die Grund-

16 Weber \& Fischer 2012, S. 2.

17 Siehe dazu auch die Einleitung. Genaue Zahlen dazu sind schwierig zu erheben, ein Indikator für Österreich ist die Zahl der Hofsuchenden, die bei der Vermittlungsplattform »Perspektive Landwirtschaft« anfragen: Waren es im Jahr 2018 noch 82, hatten im Jahr 2019187 Einzelpersonen, Paare oder Familien ein Inserat für eine Hofsuche geschaltet. (Persönliche Information am 1.7.2020 der Geschäftsführung, https://www.perspektive-landwirtschaft.at). 
nahrungsmittel für Menschen produziert, während sie gleichzeitig den Lebensmittelproduzent*innen materiell, sozial und umweltethisch zufriedenstellende Lebensbedingungen ermöglicht. An welches überlieferte Wissen knüpfen die »neuen Bäuerinnen und Bauern« an und welches Wissen bringen sie mit? Schließlich: Wie generieren sie das (neue) Wissen, das sie für die Bewirtschaftung ihrer Höfe benötigen und wie entwickeln sie neue Techniken und Organisationsformen?

Die Vermarktungsmöglichkeiten für bäuerliche Produkte waren im Laufe des 20. Jahrhunderts ${ }^{18}$ bis in die Gegenwart ebenso veränderlich wie die zur Verfügung stehenden Technologien und die Ansprüche der Konsument*innen. Unsere zentrale These lautet daher: Neueinsteiger*innen und Quereinsteiger*innen entwickeln Produktionsweisen und erschließen Vermarktungswege, die es bislang so noch nicht gab. $^{19}$

Für die Auswahl der Fallbeispiele für unser Forschungsprojekt haben wir folgende Kriterien festgelegt: Haupterwerbsbetriebe, die zertifiziert-ökologische Grundnahrungsmittel erzeugen. ${ }^{20}$ Im Sinne einer »maximalen Kontrastierung« zur Gesamtbewegung in der Landwirtschaft - nämlich der Schließung von Betrieben - suchten wir als ersten Betrieb einen neu gegründeten Betrieb aus. ${ }^{21}$

\section{Eduard und Raphaela (Fall Adam): Von der Magerwiese zur intensiven Gemüsewirtschaft}

Eduard und Raphaela gründen im Jahr 2015 einen Öko-GemüsebauBetrieb. Ihre beiden Kinder sind gerade neun und drei Jahre alt. Sie

18 Siehe dazu ausführlich das Kapitel von Elisabeth Kosnik.

19 Auch der agrarische Ausblick der Landwirtschaftskammer stellt fest: »Die Gruppe der Neu-Einsteiger in die Landwirtschaft ist zwar sehr klein, kann aber in Bezug auf Öffentlichkeit und Ideenreichtum viel zur Gesamtentwicklung beitragen.«Siehe Österreichische Landwirtschaftskammer 2016:15.

20 Daher haben wir zum Beispiel Imkerei-Betriebe oder Kräuterbetriebe ausgeschlossen.

21 Zur Fallauswahl siehe Methodenkapitel. 
wandeln ca. $8.000 \mathrm{~m}^{2}$ Wiese in Gemüseland um. Das Land gehört Raphaelas Eltern und wird seit ca. 30 Jahren sehr extensiv und biologisch bewirtschaftet. Die Böden sind karge Sandböden. Rund um das Wohnhaus liegen einige Nebengebäude, die als Lagerräume dienen, sowie Wohnwägen - als Wohnmöglichkeit für die im Sommer zahlreichen Mitarbeiter*innen (WWOOFer*innen, ${ }^{22}$ Praktikant*innen, Lehrlinge). Eduard leitet den Gemüsebau, Raphaela die Vermarktung. Sie vermarkten ihr Gemüse auf zwei Wochenmärkten nahegelegener Kleinstädte, liefern an vier Foodcoops ${ }^{23}$ und ein Catering-Unternehmen sowie einen Reformladen in Wien. Zum Zeitpunkt des Interviews arbeiten sie gerade am Aufbau einer Community Supported Agriculture (CSA). ${ }^{24}$ Eduard bietet regelmäßig Workshops zum Anbau von Bio-Gemüse auf ihrem Betrieb an.

Mit 17 Jahren kommt Eduard aus seinem Geburtsland Mali nach Frankreich, wo er eine Landwirtschaftsschule und eine Gartenbauschu-

22 World Wide Opportunities on Organic Farms - freiwillige Helfer"innen auf ökologischen Höfen (www.wwoof.at).

23 Eine Foodcoop ist ein Zusammenschluss von Einzelpersonen und Haushalten, die selbstorganisiert Lebensmittel direkt bei Bauern, Gärtnereien oder Imkern aus der jeweiligen Region beziehen. In den letzten Jahren sind in Österreichvor allem in Städten, aber auch in einigen Landgemeinden - Foodcoops entstanden. Im Jahr 2020 gab es in Österreich 91 Foodcoops (www.foodcoops.at, abgerufen am 5.6.2020). Siehe auch Heistinger 2018.

24 Auch unter der Bezeichnung Solidarische Landwirtschaft (SOLAWI) bekannt. Basis ist eine Art Vertragslandwirtschaft: Eine Gruppe von Menschen nimmt die Erzeugnisse einer Gärtnerei/eines landwirtschaftlichen Betriebes verbindlich ab und deckt mit diesen fixen Beiträgen die Ausgaben der Betriebsführung - und sichert so auch das Einkommen für die ProduzentInnen. Durch einen vielfältigen Anbau kann ein CSA-Betrieb dutzende bis einige hundert Menschen über einen langen Zeitraum mit frischem Gemüse, Obst und Kräutern versorgen - manche schaffen sogar eine Canzjahresversorgung. Eine CSA ist eine Nutzen- und eine Risikogemeinschaft: Die vorhandene Ernte wird auf alle Mitglieder der CSA gleichmäßig aufgeteilt. Ist die Ernte gering-zum Beispiel aufgrund von Trockenheit oder Hagel - ist auch jeder Ernte-Anteil kleiner (wobei der Kosten-Anteil gleich bleibt). Das Konzept bietet vor allem neu gegründeten Betrieben einen ökonomischen Start-Vorteil. Siehe ausführlich Heistinger 2018, S. 136-165. 
le besucht. An dieser Schule lernen sich Eduard und Raphaela - die an der Schule ein Austauschsemester absolviert - kennen. Ein Lehrer der Französischen Schule organisiert für ihn ein Praktikum auf einem biologisch-dynamischen Gemüsebaubetrieb in Österreich, wo er ab dem Jahr 2005 auch für einige Jahre arbeitet, bevor er zunächst mit einem befreundeten Bauern und dann mit Raphaela einen eigenen Betrieb gründet. Raphaela ist die jüngste von vier Geschwistern. Sie ist Akademikerin und studierte Internationale Entwicklung.

Weder Eduard noch Raphaela haben den Anbau von ökologischem Gemüse in ihren Familien gelernt. Das Wissen, das sie benötigen, um Gemüse ertragreich anzubauen ist daher kein überliefertes Erfahrungswissen. Ebenso wenig hat der Erwerbsanbau von Gemüse in der Gemeinde, in der der Betrieb liegt, eine Geschichte. Hingegen erschließt sich eine Ressource für die Idee einen Gemüsebau-Betrieb in einer trockenen Region zu gründen und diese Idee auch in die Tat umzusetzen aus den Familienbiografien von Eduard und Raphaela: Sowohl Eduards Großvater wie auch sein Vater waren im westlichen Sahel für das größte Bewässerungsprojekt der französischen Kolonialmacht tätig. Sein Großvater als zwangsrekrutierter Bauer, sein Vater viele Jahre später als leitender Angestellter der Baumwollindustrie - nach einer Ausbildung zunächst in Mali, später in Frankreich. ${ }^{25}$ Die Technik der Auseinandersetzung mit Klima und Region wird aus dieser biographischen Erfahrung Eduards her erklärbar. Der Gewissheit, dass Pflanzen auch in einer Trockenregion Österreichs ertragreich wachsen können, folgt die Fähigkeit die regionale Bodenbeschaffenheit - und wie die Pflanzen darauf reagieren - zu beobachten und zu erspüren. Diese Techniken des Wahrnehmens, etwa was Gemüsepflanzen für eine ertragreiche Ernte benötigen, begünstigt somit unabhängig von der Region den bäuerlichen Erfolg und generiert neues Wissen. Diese familiäre Kompetenz kombiniert Eduard mit seiner Ausbildung und dem Wissen und der Erfahrung aus seiner mehrjährigen Anstellung auf einem ökologischen Gemüsebaubetrieb in der Region.

25 Diese Ausbildung hatte ihm der auch in Mali tätige Französische Don BoscoOrden ermöglicht. 
In der Familiengeschichte Raphaelas fällt auf, dass es in der Familie ihrer Großmutter Erfahrungen des Verlustes von Grund und Boden, des sozialen Abstiegs und des sich in einem neuen Land neu sozial und ökonomisch zu verwurzeln gibt sowie eine hohe Bildungsorientierung. Diese Erfahrungen verdichten sich in der Familie zum Muster »Als Familie unkonventionelle Lebensentscheidungen zu treffen«. In Bezug auf die persönliche Resilienz-Potentiale der beiden fällt auf, dass sowohl Raphaela wie auch Eduard bewusst an die Ressourcen aus ihren Familien anknüpfen. Eduard beschreibt die Geschichte seines Vaters und seines Großvaters als Erfolgsgeschichte. Auch Raphaela nimmt viele Erfahrungen und Kompetenzen ihrer Familie als Ressourcen wahr und beschreibt diese in den Gesprächen ausführlich und wertschätzend. Dieses positive und stärkende Anknüpfen an die eigenen Familienbiografien ist ein wichtiges Kapital für die Gründung eines Familienbetriebs und den Schritt in die landwirtschaftliche Selbständigkeit.

Die Betriebsneugründung von Eduard und Raphaela ermöglicht den beiden ihre jeweiligen familiären Handlungsmuster zu verweben - oder anders gesagt: in der Betriebsgründung kommen beide familiären Handlungsmuster zum Ausdruck. Zusätzlich bringen beide auch unterschiedliche Ressourcen für die Gründung des Betriebes mit: Während Eduard - durch seine Ausbildung und seine berufliche Tätigkeit vor der Betriebsgründung - das pflanzenbauliche Wissen mitbringt, bringt Raphaela das Land ein, auf dem der Betrieb angelegt werden kann, sowie Offenheit, Vertrauen und Mut, sich auf neue Lebenskonzepte einzulassen und einen landwirtschaftlichen Betrieb als Lebensgrundlage ihrer jungen Familie zu gründen (zum Zeitpunkt der Betriebsgründung sind ihre beiden Kinder bereits auf der Welt). In die Ausrichtung des Betriebs - Anbau von ökologischem Gemüse, Direktvermarktung und das Anbieten von Seminaren - fließen verschiedene Ressourcen aus beiden Herkunftsfamilien mit ein.

Im Sinne der maximalen Fallkontrastierung wurde als zweites Fallbeispiel ein Betrieb ausgewählt, der innerhalb der Familie übernommen wurde. 


\section{Martin und Andrea (Fall Bergmann): Vom Weltmarkthuhn-Betrieb zur Öko-Gemüse CSA}

Martin und Andrea bewirtschaften ihren ca. drei Hektar großen Betrieb seit dem Jahr 2007. Auf dem Betrieb leben sie mit ihren beiden Kindern, die 2015 und 2017 auf die Welt gekommen sind, sowie mit Martins Mutter. Auf rund 1,5 Hektar bauen sie Gemüse und Kartoffeln an. Der Betrieb hat eine umfangreiche Ausstattung an Gebäuden. Martin hat den elterlichen Betrieb sehr jung übernommen, nachdem sein Vater erkrankt und verstorben war. Bis zum Jahr 2012 war der Betrieb ein intensiver Geflügelbetrieb, der Bruteier für die Mast-GeflügelProduktion erzeugte. Die von einem holländischen Zuchtunternehmen angelieferten Küken wurden aufgezogen, ihre Eier abgenommen und ausgebrütet. ${ }^{26}$ Martin und Andrea erzählen im Interview: »Der Knackpunkt war eher der, dass wir gesagt haben, so kann es nicht weitergehen. In diese Richtung wollen wir nicht weitermachen. Und dann haben wir Alternativen gesucht. «Sie entschieden sich die Intensiv-Hühnermast zu beenden. Die erste Überlegung war, dass Martin eine Erwerbstätigkeit außerhalb der Landwirtschaft annehmen könnte. Schließlich brachte Andrea die Idee, wie sie den Betrieb stattdessen bewirtschaften können, aus ihrem Studium der Umweltpädagogik mit. Auf einer Exkursion hatte sie die erste Community Supported Agriculture Österreichs kennen gelernt. Andrea: »Und so ist die Idee dann eigentlich entstanden, dass wir das bei uns ja auch machen können. «Für Martin war diese Idee anfangs »ganz weit weg«. Eindrücklich beschreibt er die Transformation seines eigenen BetriebsVerständnisses: »Für einen Agraringenieur, der als Landwirtschaftsmanager ausgebildet worden ist, ist ein System, wo die Betriebswirtschaft ein bisschen in den Hintergrund rückt und andere Faktoren entscheidender sind, das ist nicht so gleich vorstellbar gewesen. «Sein innerer Transformationsprozess, erzählt

26 Die Legehennenzucht ist eine der intensivsten Tierhaltungen der industriellen Landwirtschaft. Die Hühner sind Hybridrassen, weltweit gibt es drei Konzerne, die diese Legerassen züchten und die Tiere werden über weite Strecken transportiert. Teil dieser Tierindustrie ist auch das Töten der männlichen Küken, da diese in diesem System »überflüssig« sind. 
Martin, habe aber bereits davor begonnen: Im Sterbejahr seines Vaters habe er mit diesem noch ein neues Wirtschaftsgebäude - für den Ausbau der Hühnerhaltung - errichtet. Für seinen Vater sei dies eine "perfekte Betriebssituation « gewesen, wie »er es sich vermutlich erträumt hat «. Ihn hingegen habe es »aber nicht befriedigt oder nicht genug das Feuer in mir genährt«. Es habe keinen fachlichen oder betrieblichen Grund gegeben, die Produktion von Bruteiern aufzugeben: »Das war eine Nische und wenn du da gut warst, hast du gut damit verdient, keine Frage." Einige Jahre nach dem Tod seines Vaters erlebt Martin ein Burnout. Er habe sich einfach nicht mehr motivieren können, in den Stall zu gehen: »Ich hab einfach nicht mögen, das war so eine müde Zeit. « Genau in dieser Krise Martins absolviert Andrea ihr Studium der Umweltpädagogik und kommt mit neuen Ideen, einem anderen Naturverständnis und dem konkreten Vorschlag, einen CSA-Betrieb zu gründen, nach Hause. Noch im gleichen Sommer - des Jahres 2011 - besuchen die beiden andere Höfe als Inspiration. Einen Betrieb in Oberösterreich, der seit fast 40 Jahren Gemüse für die Selbstversorgung und die Direktvermarktung anbaut und neue Anbautechniken (Mischkultur und Permakultur) entwickelt hat, sowie von Kolleg*innen gegründete CSA-Betriebe. Die Kontakte zu ihnen stammen von Andrea über ihr Studium. Auf die Frage, wie er sich das gemüsebauliche Wissen angeeignet hätte, meint Martin: »Ich habe den ersten Fenchel angebaut, bevor ich ihn jemals selber gegessen habe." Grundsätzlich wären sie gerne für eine Saison als Praktikant*innen auf einen anderen Betrieb gegangen. Das würden er Neu-Einsteiger*innen empfehlen, meint Martin: »Wenn wir die Zeit gehabt hätten, wäre es schön gewesen auf einen anderen Gemüsebaubetrieb zu gehen (...), ich sehe das als gutes Fundament, um dann selbst später einen Betrieb aufzumachen. «Überhaupt meint er, dass gerade für den Anbau von Gemüse in Form eines CSABetriebes die Praxis besonders wichtig sei:

»Das glaube ich nicht, dass das über die Fachschulen laufen kann, sondern wenn, dann nur über die Betriebe, wenn man sie wirklich hautnah erlebt. Weil es ist ein totaler Spezial Know-How-Bereich. Und die Innovationen passieren aufden Betrieben und nicht in den Schulen." 
Im Jahr 2012 stellen Andrea und Martin den Betrieb auf Bio um, im Jahr 2013 starten sie mit dem Konzept als CSA-Betrieb. Als eine essentielle Ressource, um von der industriellen Hühner-Produktion aussteigen zu können, benennt Martin die niedrige Schuldenbelastung des Betriebs: »Wenn wir nicht diese niedrige Schuldenbelastung gehabt hätten, hätten wir es uns gar nie leisten können, aus dem Auszusteigen."

Die Entscheidung der beiden Betriebsleiter*innen, die intensive Hühnerhaltung aufzugeben und auf einen CSA-Betrieb umzustellen, erscheint aufs erste als radikaler Bruch mit der Geschichte des Betriebs. Bei einer sequentiellen Analyse des Genogramms der Eltern und Großeltern der Betriebsleiter*innen wird hingegen sichtbar, dass das SichEinlassen-Können auf neue Wirtschaftsweisen und das Eröffnen neuer Betriebszweige sowie die Weitergabe des eigenen Erfahrungswissens und der Erfahrungs-Austausch mit anderen Betriebsleiter*innen ein Muster ist, das auch für die Eltern- und Großelterngeneration von Martin zutrifft. Auch sie konnten sich jeweils als erste in der Region auf radikal Neues einlassen - den Anbau damals neuer Kulturen, neuer Anbaumethoden sowie neuer Vermarktungswege. Durch die familiäre Offenheit für neue Ideen und Bewirtschaftungsweisen entstehen neue Perspektiven für die Bewirtschaftung des Betriebes, neue Einkommensmöglichkeiten für den Betrieb und diese stärken die eigene Arbeitsfähigkeit und Motivation. Bedeutsam für die Transformation des Betriebes waren die Erfahrungen und Kontakte, die Andrea durch ihre Ausbildung mit auf den Betrieb gebracht hat. Sie selbst bringt kein direktes landwirtschaftliches Wissen auf den Betrieb mit - ihr Vater ist Handwerker, ihre Mutter Büro-Angestellte - doch auch ihre Großeltern waren Bauern und Bäuerinnen. Möglicherweise ist es auch diese Kombination, die sie einen frischen und unvoreingenommenen Blick auf den Betrieb und die Möglichkeiten, die sich hier bieten, einnehmen lässt.

Als maximale Kontrastierung zum zweiten Fallbeispiel wählten wir einen ökologischen Betrieb, der neu gegründet worden war. 


\section{Marianne und Heinz (Fall Conrad): Von der Lohnarbeit zur Landwirtschaft}

Marianne und Heinz kaufen den bereits aufgelassenen landwirtschaftlichen Betrieb im Jahr 2002 und gründen einen ökologischen Ziegenund Kuhbetrieb mit eigener Hofkäserei und Direktvermarktung. Der Betrieb umfasst 20 Hektar, 160 Ziegen und 30 Stück Kühe und deren Nachzucht. Marianne und Heinz stammen aus einem westlichen Bundesland Österreichs und haben Berufe außerhalb der Landwirtschaft erlernt. Marianne ist gelernte Hotelfachfrau und Heinz Maschinenschlosser. Die Familie hat an ihrem damaligen Wohnort mit der Ziegenhaltung angefangen: »Wir haben zuerst zwei Ziegen gehabt, dann haben wir 13 Ziegen gehabt und dann haben wir noch Kühe dazu gehabt und dann hat sich das Ganze entwickelt«, beschreibt Marianne den Ausbau ihrer landwirtschaftlichen Tätigkeit. Im alpinen Berggebiet war die Aussicht auf einen eigenen Hof jedoch gering. In der lokalen Zeitung sei ein Hof in der Oststeiermark inseriert gewesen. »Wir haben ja überhaupt nicht die Steiermark ausgesucht. Wir wären eher so Kanada, Australien - so in diese Richtung gewesen «, erzählt Marianne über ihre Pläne. Sie suchten nach einem Hof, der aus einer zusammenhängenden Fläche bestehe, maschinell bearbeitbar sei - vor allem für die Heuarbeit - und keine Nachbarn hätte. Wegen der Weidehaltung der Ziegen hatten sie zu Hause schon schlechte Erfahrungen mit Nachbarn gemacht. Sie wollte die Tiere im Freien halten, daher war die Abgelegenheit des Hofes für sie ein großes Kriterium. Sie konnte sich nicht vorstellen, die Tiere nur im Stall zu halten:

»Da muss ich es so machen, wie die Großen. Da muss ich sagen, okay, ich stell einen Großbunker hin, alle Tiere bleiben brav drinnen und ich tu so großbauernmäßig. Das ist für mich nicht die Landwirtschaft, die ich mir vorstelle. Wir haben uns eigentlich für Bio entschieden, weil ich einfach für artgerechte Tierhaltung bin. Und auch für Wertschätzung für Natur und Tier.«

Da sie für die Hofbesichtigung die Tiere nicht alleine hätten lassen können, sei ihr Mann damals alleine in die Steiermark gefahren: 
"Dann ist mein Mann hergekommen und hat sich das angeschaut und hat dann geschrieben, das ist super, das kann man alles mit dem Traktor fahren, das ist voll klass. Acht Tage später waren wir mit Sack und Pack und Finanzierung da. Also gesucht, gefunden, gekauft. Das gibt es ja sonst nicht."

Der Prozess der Wissens-Aneignung über die Käse-Herstellung war für Marianne intensiv und erstreckte sich über viele Jahre. Bereits an ihrem alten Wohnort habe sie begonnen, sich damit zu beschäftigen. Zunächst besuchte sie verschiedene Kurse. Nicht nur in ihrer unmittelbaren Nähe, sondern auch in Deutschland und der Schweiz. An der zu ihrem Wohnort nächstgelegenen Ausbildungsstätte hätte sie damals keinen Kursplatz bekommen. Hier wäre sie nur aufgenommen worden, wenn sie bereits in einer Sennerei angestellt gewesen wäre. Dazu meint sie im Interview kritisch: »Aber dann brauch ich ja den Kurs nicht, dann brauch ich ja die Landwirtschaftsschule nicht machen, dann hätte ich sie (Anmerkung: die Kompetenz dazu) ja schon. "Marianne war von Anfang an sehr genau und hat viel Zeit in das Erlernen des Käsens investiert. Sie meint: »Weil ich denke mir, entweder macht man es ordentlich oder gar nicht. "Zudem habe sie von den verschiedenen Käserei-Kursen sehr profitiert und für sich und die Vielfalt an verschiedenen Käsen, die sie anbietet, viel herausholen können. "Und außerdem ist es auch interessant, weil die Schweiz ist in Punkto Käsemachen ein Hauseck anders als Österreich, und auch die Bayern. Es ist ein Unterschied zwischen Vorarlberg und Steiermark, komplett anders«. Zusätzlich zu den Kursen ist für Marianne gute Fachliteratur wichtig. Es gäbe am Markt sehr viel schlechte Literatur zum Thema Käsen, bei denen bei den Rezepten kleine Fehler enthalten wären, die aber dann auf das Endprodukt eine große Auswirkung hätten. Auf die Frage, wie sie die Qualität guter Fachliteratur ausmache, meint sie: »Das siehst du sofort am Rezept. Jemand, der Kuchen backt, liest das auch sofort. Wenn ich Kuchen backe, weiß ich, das funktioniert so nicht, das muss ich ein bisschen umändern. "Zusätzlich zu den Kursen sei zu Beginn für sie wichtig gewesen, dass sie sich bei einem Bekannten ihres Mannes immer wieder Tipps holen hätte können: »Die praktischen Tipps, die kannst du dir halt auf keinem Kurs holen." Besonders wichtig sei auch gewesen, dass er ihr nicht nur Angaben - zum Beispiel über die Zeit, wie lang man den Käsebruch 
rühren müsse - gemacht habe, sondern konkret gezeigt hätte, wie sich die Käsemasse nach einem bestimmten Arbeitsschritt anfühlen müsse: »So muss sich jetzt der Druck anfühlen, dann passt das«. Gerade auf den Käsereikursen in Österreich sei es - im Gegensatz zu Deutschland - üblich, dass die Referent"innen »entweder nicht vermitteln könnten oder etwas bewusst zurückhalten« würden.

Marianne hat zunächst begonnen, ihre Käse an verschiedene Hotels und Gastwirtschaften in der Nähe ihres neuen Hofes zu vermarkten. Nach ein paar Jahren ist sie wieder davon abgekommen, da sie sich hier nicht frei fühlte: »Du bist dann auch wieder in diesem System drinnen, (...) du bist gezwungen, das zu machen, was man dir sagt. Das möchte ich nicht.«Die Einschränkung durch die fixen Speisekarten der Hotels sei ihr zu groß gewesen. Mittlerweile vermarktet sie den Käse zu $100 \%$ direkt und zwar auf vier verschiedenen Bauernmärkten in der Landeshauptstadt. »Ich möchte einfach kreativ sein und meine Sachen machen. Und das kann ich halt, wenn ich direkt verkaufe, besser «. Besonders ausführlich erzählt Marianne, wie wichtig es für ihre Produktion ist, dass sie direkt im Kontakt mit den Kund"innen ist: "Du kannst es dem Kunden erklären und weißt sofort, was er will und bekommst sofort eine Rückmeldung und du weißt, passt es oder passt es nicht. "Auch auf das Wetter und die damit zusammenhängenden Essgewohnheiten könne sie gut reagieren:

»Du weißt, jetzt ist Sommer, jetzt brauch ich das und das. Man bekommt ein Gefühl dafür. Gerade mit meinen Produkten kann ich ja schneller etwas herzaubern. Mach ich halt im Winter Nuss und Kürbis. Im Sommer mach ich halt dann eben Grillkäse, Halloumi-mäßig. Was einfach speziell auf das Wetter passt.«

Auch »gewinnmäßig« sei die Direktvermarktung das Beste für den Betrieb.

Das Fallbeispiel der Familie Conrad zeigt eindrücklich, welche Ressourcen Menschen mitbringen (müssen), um einen landwirtschaftlichen Betrieb mit Tierhaltung aufbauen zu können. Neben der Liebe zu Tieren ist dies die Fähigkeit und die Bereitschaft beharrlich und kontinuierlich zu arbeiten und für die Versorgung der Tiere Verantwortung zu übernehmen. Da die Tierhaltung mit höheren Investitionskos- 
ten verbunden ist als der Gemüsebau (wie im ersten Fallbeispiel), war es für die beiden Betriebsgründer*innen essentiell, dass Heinz von Beginn an in der Region in seinem erlernten Beruf einen Arbeitsplatz hatte denn nur so war es überhaupt möglich, von der Bank einen Kredit für den Kauf des Hofes zu bekommen. ${ }^{27}$ Dass Heinz nun gemeinsam mit seiner Frau den Hof betreibt, ist - aus seiner Familiengeschichte heraus - als sozialer Aufstieg zu verstehen. Marianne wiederum bringt aus ihrer Familiengeschichte - und vermutlich vor allem von ihren weiblichen Rollenvorbildern - ein Arbeitsverständnis mit, das einerseits von einer starken Kundenorientierung geprägt ist, anderseits von der Fähigkeit, eigene Wege $\mathrm{zu}$ gehen und $\mathrm{zu}$ improvisieren. Sie scheint es gewohnt zu sein, ihren Weg auch gegen Widerstände (der Behörden, von Kolleg*innen) zu gehen und diese zu überwinden. ${ }^{28}$ Ein möglichst selbstbestimmtes bäuerliches Leben und möglichst autonomes Handeln ist ein Handlungsmuster, das beiden wichtig zu sein scheint. Eine große Unabhängigkeit ist Marianne wichtig, auch wenn viel zu Arbeiten der Preis dafür ist. Wie ihre Mutter als Frisörin und ihre Großmutter als Schneiderin lebt Marianne gewissermaßen von »ihrer Hände Arbeit«. Möglicherweise haben beide keine familienbiografische Vorlage dafür, dass es für den Aufbau eines Betriebs auch eine Möglichkeit - und für bestimmte biografische Momente möglicherweise auch eine Notwendigkeit ist - Mitarbeiter*innen am Betrieb zuzuziehen. So ist zur Zeit die - gerade erst 13 Jahre alte Tochter - die einzige, die das Käsen übernehmen könnte, sollte Marianne ausfallen. Dies könnte zu einem Risiko

27 Bätzing betont, dass Erwerbskombinationen für die Stärkung der Regionalwirtschaft grundlegend sind, weil sie die Flexibilität des ländlichen Wirtschaftens ermöglichen. Ohne erreichbare Nebenerwerbsmöglichkeiten wären die Betriebe gezwungen, nach agrarindustriellen Bedingungen für den globalen Markt zu produzieren. Bätzing 2020, S. 236-241. Auch die Agrarsoziologinnen Heide Inhetveen und Mathilde Schmitt sehen in der Erwerbskombination die Möglichkeit für Menschen, die einen bäuerlichen Betrieb im Nebenerwerb führen, ihre bäuerliche Orientierung aufrecht zu erhalten und bezeichnen dies als Merkmal einer bäuerlichen Überlebenskultur. Siehe dazu Inhetveen und Schmitt 2010:111.

28 Siehe dazu auch das Kapitel von Elisabeth Kosnik. 
für die Entwicklung des Betriebes werden, wenn es nicht gelingt, familienfremde Arbeitskräfte einzubeziehen.

Als viertes Fallbeispiel wählen wir einen Betrieb aus, der wiederum in der Familie übernommen, aber bereits von der Elterngeneration auf ökologischen Landbau umgestellt wurde.

\section{Daniela und Anton (Fall Danninger): Vom ererbten Hobbybetrieb zum Haupterwerbsbetrieb}

Daniela und Anton bewirtschaften einen Betrieb, der seit dritter Generation in Familienbesitz ist. Antons Großeltern kaufen den Betrieb in den 1960er Jahren und führen ihn von Wien aus. Zu dieser Zeit hat der Betrieb in erster Linie als Sommer-Landsitz der bürgerlichen Familie eine Bedeutung. Nach eigenen »Bio-Versuchen« Mitte der 1980er Jahre übernehmen Antons Eltern den Betrieb und stellen ihn auf die ökologische Wirtschaftsweise um. Beide Eltern waren bereits von der ökologischen Wirtschaftsweise überzeugt. Auch sie bewirtschaften den Betrieb im Nebenerwerb und von Wien aus, wo sie beide berufstätig sind. In seiner Kindheit verbringt Anton, der in der Großstadt aufwächst, viele Wochenenden und Sommerferien am Betrieb. "Das war immer lässig«, erzählt er. Im Jahr 1985 haben die Eltern einen schweren Autounfall - Anton ist zu diesem Zeitpunkt erst 17 Jahre alt. »Da musste ich sowieso einspringen. Meine Schwester auch «, erzählt Anton. Da er in dieser Zeit gemerkt habe, dass ihm die »Arbeit am Hof recht gut gefällt«, habe er sich nach der Matura entschieden, eine Ausbildung zum landwirtschaftlichen Facharbeiter zu machen. Vor der Umstellungsphase hätte er zwei Vergiftungen durch ein Insektizid gehabt und daraufhin den ersten ökologischen Obstbaubetrieb Österreichs besucht. Hier lernt er auch seine erste Partnerin Isabella kennen, die im Zuge ihrer ObstbauAusbildung hier ein Praktikum macht. Isabella, die von einem landwirtschaftlichen Betrieb stammt, studiert Biologie. Im Jahr 1992 kommt ihre erste Tochter zur Welt, 1996 der erste und 2000 der zweite Sohn. Erst im Jahr 1996 übernimmt Anton den elterlichen Betrieb - der damals verschuldet war - und Anton und Isabella, die wie er vom biologischen Landbau überzeugt ist, stellen ihn auf Vollerwerb um. 
Aktuell umfasst der Hof mit zugepachtetem Boden rund 7 ha Obstbaufläche. Das Obst (Äpfel, Birnen und Quitten) wird lokal über einen Bauernladen vermarktet, sowie über Bioläden, ein Seminar-Hotel, ÖkoGroßhändler und eine österreichische Supermarkt-Kette. Daniela, Antons spätere Frau, die ebenso wie Isabella Biologie studiert hat, arbeitet im lokalen Naturpark und bietet Eselwanderungen an. Bei der Ernte helfen "Pensionisten und Hausfrauen « aus der Umgebung, die sehr verlässlich seien und die Arbeit gerne machen würden: »Man weiß genau, wo sie sind, weil sie so viel lachen (...) und die hört man durch die ganze Anlage«. Anton erzählt, dass er froh sei, dass er »die Ernte nicht busweise organisieren « müsse - womit er meint, keine Ernte-Arbeiter"innen aus dem benachbarten Ausland organisieren müsse. Wobei Daniela erzählt, dass ihre Erntehelfer*innen schon älter seien und kaum jüngere aus der Gegend nachkommen würden. Überhaupt fehle am Hof »die Elterngeneration«, die bei der Arbeit unterstützen könne. Eine große Erleichterung sei neuerdings die Mitarbeit des jüngsten Sohnes Lorenz.

Anton kann kaum auf familiäres Know-How zum Obstbau zurückgreifen. Er erzählt: »Praktisch als nicht am Land aufgewachsener, ganz normaler Maturant hatte ich von Landwirtschaft null Ahnung. Ja, es war ein Hineinwachsen. Meine Oma ist leider zu früh verstorben, um ihr Wissen weiterzugeben. Ah, das war verdammt viel Lehrgeld, viel ausprobieren, viel fragen. Ich hatte gottseidank ein paar Freunde (...), die mir viele Sachen gezeigt haben, erklärt haben, und dann ist es wirklich ausprobieren und versuchen. « Zum BioObstbau habe er sich sein Wissen aus ganz unterschiedlichen Quellen angeeignet: Auf den ersten Ökologischen Obstbautagungen in Deutschland, durch den Austausch mit andern Bio-Obstbauern aus der Region und aus anderen Regionen Österreichs, sowie aus Deutschland. Später erst sei er auch von einem von der Landwirtschaftskammer finanziertem Berater für ökologische Landwirtschaft beraten worden. Ein Unterschied zu jener Zeit, in der sie mit dem Bio-Obstbau begonnen hätten, sei, dass es nun kaum mehr einen Spielraum für Versuche gebe: »Damals gab es ja auch nicht viel Forschung zum Thema biologischer Obstbau, da haben wir einfach alle probiert, was gegangen ist." Anton weist auch darauf hin, dass es nun in Österreich, Deutschland, Südtirol und Slowenien viele Versuchsanstalten gebe, die sich auch mit dem biologischen 
Anbau beschäftigen würden. »Man kann sich aus allen (...) etwas herauspicken.« Jedoch sei der Unterschied: »Du bist dann reiner Produzent (...), wirtschaftlich optimierter Produzent. Das ist der Unterschied zu damals, wo du dich noch selber gespielt hast.«

Bei der Übernahme sei der Betrieb »hoch verschuldet « gewesen und auch Investitionen seien zu tätigen gewesen. Anton erzählt, er habe »nur übernommen«, damit der Betrieb »nicht verkauft« wird. »Es ist ein Mordsstress, eben diese Wirtschaftlichkeit, aber es fängt an, sich wirtschaftlich zu rechnen. Mit dem negativen Aspekt, dass es irgendwie (...), der Reiz der Landwirtschaft dem wirtschaftlichen Aspekt untergeordnet wird. «Daniela erzählt, dass sie und ihr Mann »um jeden Millimeter « feilschen würden zwischen Wirtschaftlichkeit und technischen Fragen des Obstbaus einerseits (Antons Interesse) und andererseits anderer Qualitäten des Betriebes, wie Lebensräume für Wildtiere oder nicht unmittelbar produktive Pflanzen und Tiere am Betrieb (Danielas Interesse). Diese seien aber notwendig, da sie den Betrieb auch interessant machen würden für zum Beispiel Schulklassen. Daniela: »...dass ich diejenige bin, die wieder Aktionstage macht am Hof, die Schulen an den Hof bringt, die dann im Herbst so schauen, wie gepflückt wird, und eine Apfelverkostung machen (...). Das sind so die Sachen, wo ich das Gefühl habe, da kommt man wieder am Boden an."

\section{Sorgsame Landwirtschaft und Resilienz - Gemeinsamkeiten und Unterschiede der Fallbeispiele}

Die Protokollierung der Lebenspraxen von vier Ökobauern und fünf Ökobäuerinnen und die Rekonstruktion ihrer Genogramme liefert eine genaue Beschreibung ihrer spezifischen, subjektiven Antworten auf allgemeine Handlungsprobleme bäuerlicher Betriebe der Region. Jede Betriebsgeschichte und jede Familienbiographie ist Ausdruck der vorliegenden Bewährungsanforderungen. Jeder Fall repräsentiert unterschiedliche Modi der Problembewältigung und des Umgangs mit Krisen. Die Produktionsweise und die betriebliche Dynamik eines landwirtschaftlichen Betriebes sind ein Ausdruck der Biografien der Betriebsleiter*innen. 
Die untersuchten erfolgreichen Ökobetriebe setzen individuell gefundene Wirtschaftskonzepte um, die nicht im Sinne von Modellen generalisierbar sind. Dennoch sind aus diesen gemeinsame Handlungsmuster herauslesbar: Alle Betriebsleiter*innen zeichnet aus, dass sie bereit sind, in ihrem unmittelbaren Umfeld noch nicht bekannte - geschweige denn etablierte - Wirtschaftskonzepte umzusetzen. Alle vier Betriebe können daher als Pionier-Betriebe bezeichnet werden. Gerade in diesem Zusammenhang fällt auf, dass die Betriebsleiter*innen, die ansonsten in der Privatwirtschaft verbreitete Praxis, Wissen exklusiv für sich zu behalten, nicht pflegen und betonen, wie wichtig der Austausch mit anderen Betrieben, aber auch mit Konsument*innen und anderen Akteur*innen ist. Im Gegenteil haben sie großes Interesse, Wissen unter Kund*innen und Kolleg*innen zu verbreiten und sich in Communities auszutauschen. Möglicherweise auch deswegen, da eine Konkurrenz auf Absatzmärkten bei regionalem Vertrieb und Ablehnung des Wachstumsdogmas nicht zu befürchten ist. Ein weiterer Grund dafür mag sein, dass sie von der Qualität ihrer Produkte überzeugt sind, auf Qualität (statt Quantität) setzen und diese mit Überzeugung vermarkten. Im Rahmen kleiner regionaler Betriebe erzeugt ein non market trade Vorteile - vor allem der Austausch von Wissen und Erfahrungen über Produktionsmethoden, die die eigenen betrieblichen Ressourcen (wie die Bodenfruchtbarkeit der Betriebe) schonen oder über kostengünstige Methoden der Herstellung von Geräten, über gute Erfahrungen zu Lieferanten oder zum Aufbau von neuen sozialen Strukturen wie einer CSA, die viel betriebswirtschaftliches Organisationswissen - etwa wie man eine neu entstehende Gemeinschaft organsiert - benötigt. Dieses Wissen entsteht neu auf den Betrieben. Für kleine und regionalspezifisch wirtschaftende Betriebe gilt, dass der profitfreie Austausch von Expertisen die Gewinne aller erhöht.

Drei der vier Betriebsleiter-Paare erzählen, dass für sie Freundinnen und Freunde, die ihnen landwirtschaftliches Know-How vermittelt hätten, besonders zu Beginn für sie wichtig gewesen wären. Sie konnten also ihre freundschaftlichen und familiären Beziehungen als Ressourcen für die Betriebsgründung oder für die Konzeption und das In- 
die-Welt-bringen von neuen Betriebskonzepten nutzen. ${ }^{29}$ Für Martin und Andrea ist der gute Kontakt zu den Eltern auch betriebswirtschaftlich wichtig. So hat Andreas Vater, der Schlosser ist, mit Martin gemeinsam neue Gerät konstruiert zum Setzen von Jungpflanzen in Heu - was eine große zeitliche Arbeitsersparnis bedeutet.

Alle Betriebsleiter*innen betonen, wie wichtig für sie persönlich, aber auch für die Betriebe der Austausch mit ihren Kund*innen ist, die positiven Rückmeldungen zu den Produkten stärken das Vertrauen in die eigene Kompetenz und können - da sie die eigene Handlungsfähigkeit bzw. das Vertrauen in die eigene Handlungsfähigkeit stärken auch als Praktiken der Resilienz bezeichnet werden.

An die eigene Geschichte anknüpfen, an die eigene Biografie anknüpfen, an die Biografie des Ortes, das sind resiliente Handlungsmuster. Sorgsame Landwirtschaft knüpft an und verbindet, lässt los, interpretiert neu, kann sich anpassen, ist reaktionsfreudig. An die eigene Biografie anzuknüpfen heißt immer auch einen Bezug zu schaffen zu einem Kollektiv: Zur eigenen Herkunftsfamilie und ihrer Geschichte oder zum sozialen Milieu, in dem man aufgewachsen ist - dazu zählen neben den Eltern, Großeltern und Verwandten auch andere Akteur*innen. Regionale Muster der Daseinsbewältigung ${ }^{30}$ und wie sich die eigenen Eltern und Großeltern darin eingerichtet haben, welchen Handlungsmustern ihre beruflichen und betrieblichen Entscheidungen gefolgt sind, all das sind Ressourcen, an die die Betriebsleiter*innen anknüpfen können. Die Betriebsleiter*innen knüpfen so unmittelbar an der eigenen Geschichte an, an ihrer Wissensgeschichte und verwandeln sie in etwas Neues. So können sie auch neue Wissensbestände und neue Erfahrungen aus ihren Ausbildungen oder aus dem Austausch mit Kolleg*innen in das eigene Tun einweben. Gleichzeitig führt so neues Wissen nicht weg vom eigenen Wissen und von der eigene Geschichte vom Eigenen überhaupt - sondern wird zu einem eigenen Pol, der die

29 Zu ähnlichen Ergebnissen kommt auch eine Untersuchung zur Betriebsübergabe außerhalb der Familie. Siehe: Heistinger 2012.

30 Siehe dazu das Kapitel »Sozialen Sinn verstehen durch rekonstruktive Genogrammarbeit«. 
handelnden Menschen sogar wieder neu in die eigene Geschichte hineinführen kann. An die eigene Biografie anzuknüpfen, die Ressourcen aus der eigenen Familiengeschichte neu beleben zu können, führt zu Resilienz und Widerstandskraft. Dazu braucht es Offenheit und Kommunikation und Freiräume zur Reflexion über die eigene Geschichte: Was davon ist jetzt für mich und meinen Betrieb hilfreich und eine wichtige Ressource? Was ist in der alten Form nicht mehr hilfreich? Wie kann ich es neu kontextualisieren und wovon will ich mich auch abgrenzen? Die eigene Geschichte immer wieder neu zu beleben, neu zu interpretieren, bedeutet, die bisherigen Erfahrungen immer wieder neu im Moment der Gegenwart - zu kontextualisieren, in Beziehung zu gehen mit der eigenen Geschichte und der Umwelt, den Menschen aus der eigenen Vergangenheit und der eigenen Gegenwart. Dabei spielt es keine Rolle, ob die Familie in der Region, in der sich der Betrieb befindet gelebt hat oder nicht. Im Gegenteil: Die Menschen aus unseren vier Fallbeispielen - die jeweils etwas Neues in der Landwirtschaft wagten bringen Bereitschaft zu Mobilität sowie Erfahrungen des Ortswechsels aus ihrer Familienbiographie mit. Diese haben sich als wichtige Ressource für den Aufbau des Betriebes in der Region erwiesen.

Die Forschungsergebnisse zur sorgsamen Landwirtschaft sind insbesondere anschlussfähig an den Diskurs zur Agrarökologie, der innerhalb des Ökologischen Landbaus seit etwa 20 Jahren geführt wird. Diese versteht sich nicht als rein objektive Naturwissenschaft, sondern bezieht bäuerliches Wissen und Erfahrungswissen aus der Landwirtschaft mit ein. Agrarökologische Methoden sind im Vergleich zu den Methoden der industriellen Landwirtschaft wissens- und arbeitsintensiver.

Ein wesentliches Ergebnis unserer Forschung besteht in der Erkenntnis, dass es kein spezifisch lokales Wissen gibt, das an einem Ort gefunden und dort tradiert wird. ${ }^{31}$ Vielmehr zeigen die einzelnen Fälle, dass das handlungsgenerierende Wissen individuell erworben wird und von Generation zu Generation wechselt. Wissen wird erst durch das Sammeln von Erfahrungen an einem Ort anwendbar und zu embodied knowledge. Da die Herausforderungen durch Markt und 
Klima ebenso veränderlich sind wie die zur Verfügung stehenden Technologien und die Ansprüche der Konsument*innen, muss stets neues Wissen generiert werden. Überliefertes - auch konventionelles landwirtschaftliches Wissen und das Wissen über die Auswirkungen dieser Produktionsmethoden auf die eigenen Körper und Böden wird mit neuem Wissen auf kreative Weise verwoben.

Für Expertise und Handlungskompetenz werden in der Professionsforschung folgende vier Faktoren als Voraussetzung genannt: ${ }^{32}$ Umfangreiches Wissen und gutes Gedächtnis, Problemlösefähigkeit und Entscheidungsstärke, Verfügbarkeit von Routinen und die Einbettung in Expert*innengemeinschaften. Die von uns befragten Personen weisen diese Fähigkeiten auf. Eine weitere wesentliche Fähigkeit eint die Bauern und Bäuerinnen unserer Fallbeispiele: Die Fähigkeit zum Erkennen von Problemen. Oder anders gesagt: Das Neue liegt im Erkennen von Problemen, die andere so nicht sehen. Bio Landwirt"innen können Probleme nicht nur innovativ lösen, sondern haben davor aufgrund ihrer spezifischen familiären Ressourcen Faktoren verknüpft, die bisher noch nicht in dieser Konstellation erkannt wurden. Neue innovative Betriebsgründungen oder Umgründungen verdanken sich einer Kombination aus Vorwissen, Neugier, Experimentierfreudigkeit und guter Vernetzung. Dazu kommen Pflanzen oder Tiere, die in der Region gut gedeihen. Weiters braucht es die Bestärkung von Expert*innen oder anderen Menschen, denn im unmittelbaren Lebensund Betriebsumfeld wird Innovationen teils auch mit Skepsis und Ablehnung begegnet. Daher sind soziale Netzwerke - analoge wie digitale - und der überregionale Wissensaustausch für die Betriebe besonders wichtig. ${ }^{33}$ Bedeutsam ist zudem, dass die neuen Betriebsgründer*innen eine Kombination unterschiedlicher Ausbildungen und Erfahrungen vorweisen können. Wie z.B. auch Studien aus Italien zeigen, ${ }^{34}$ verfügen neue Betriebsgründer*innen über eine gute Bildung die allerdings nicht notwendigerweise eine landwirtschaftsbezogene

32 Vgl. Gruber u.a. 2006, S. 194.

33 Siehe dazu auch das Kapitel von Elisabeth Kosnik.

34 Milone \& Ventura 2019. 
Grundausbildung sein muss. Sie sind kommunikativ, können sich Information auch informell besorgen, haben fremde Länder bereist und stehen den herkömmlichen Vermarktungsformen kritisch gegenüber. Wesentliche Faktoren für die Entscheidung gegen konventionelle Landwirtschaft, die in den Gesprächen genannt wurden, waren einerseits der Wunsch nach autonomer Betriebsführung im Gegensatz zum Diktat der Weltmarktorientierung und andererseits eine Haltung der Sorge gegenüber der Umwelt. Regionale Einbettung ist nichts Stabiles, sondern ein lebendiger Zusammenhang, der durch den beschleunigten Weltmarkt noch viel veränderlicher geworden ist. 
